\title{
Gastrin-Releasing Peptide Receptor Imaging in Human Breast Carcinoma Versus Immunohistochemistry
}

\author{
Christophe Van de Wiele ${ }^{1,2}$, Philippe Phonteyne ${ }^{1,2}$, Patrick Pauwels ${ }^{3}$, Ingeborg Goethals ${ }^{1}$, Rudi Van den Broecke ${ }^{4}$, \\ Veronique Cocquyt ${ }^{5}$, and Rudi Andre Dierckx ${ }^{1,2}$ \\ ${ }^{I}$ Department of Nuclear Medicine, Ghent University Hospital, Ghent, Belgium; ${ }^{2}$ Department of Nuclear Medicine and Molecular \\ Imaging, University Medical Centre, Groningen, The Netherlands; ${ }^{3}$ Department of Anatomopathology, Ghent University Hospital, \\ Ghent, Belgium; ${ }^{4}$ Division of Gynaecological Oncology, Ghent University Hospital, Ghent, Belgium; and ${ }^{5}$ Department of Medical \\ Oncology, Ghent University Hospital, Ghent, Belgium
}

\begin{abstract}
This study reports on the uptake of $99 \mathrm{mTc}-\mathrm{RP} 527$ by human breast carcinoma and its relationship to gastrin-releasing peptide receptor (GRP-R) expression as measured by immunohistochemistry (IHC). Methods: Nine patients referred because of a clinical diagnosis suggestive of breast carcinoma and 5 patients with tamoxifen-resistant bone-mestastasized breast carcinoma underwent 99mTc-RP527 scintigraphy. The findings were compared with routine staging examinations in all patients and with routine histology and IHC GRP-R staining in the first 9 patients. All 9 patients with suspected breast lesions were tumor positive. Results: The uptake of $99 \mathrm{mTc}$-RP527 was evident in the primary tumor in 8 of 9 patients and in involved lymph nodes and part of the distant metastasis limited to the bone when present. ${ }^{99 m} \mathrm{Tc}-$ RP527 uptake was not found in any of the tamoxifen-resistant patients. Conclusion: Uptake by primary breast carcinoma was significantly correlated with the presence of GRP-Rs as assessed by means of IHC.
\end{abstract}

J Nucl Med 2008; 49:260-264

DOI: 10.2967/jnumed.107.047167

G astrin-releasing peptide/bombesin (GRP) has been shown to function as an autocrine growth factor in a variety of human tumors, including breast carcinoma (1). Its tumor growth-stimulating effect is a direct consequence of binding to the GRP G-protein-coupled receptor (GRP-R). With the aim of inhibiting breast tumor growth, this growth factor and its receptor have been evaluated as potential targets for various antagonists or blocking agents (2). As these blocking agents result in tumor stasis rather than regression, alternative techniques to morphologic imaging, assessing changes in tumor volume, will be necessary to monitor their efficacy.

Received Sep. 10, 2007; revision accepted Nov. 6, 2007.

For correspondence or reprints contact: Christophe Van de Wiele, MD, $\mathrm{PhD}$, Department of Nuclear Medicine, Ghent University Hospital, De Pintelaan 185, 9000B Ghent, Belgium.

E-mail: christophe.vandewiele@ugent.be

COPYRIGHT @ 2008 by the Society of Nuclear Medicine, Inc.
The success of ${ }^{111}$ In-pentetreotide ( ${ }^{111} \mathrm{In}$-Octreoscan; COVIDIEN, USA) in demonstrating tumors with somatostatin receptors (3) and of ${ }^{123} \mathrm{I}$-vasoactive intestinal peptide (VIP) for visualization of tumors overexpressing VIP receptors by scintigraphic imaging (4) prompted the search for a radiolabeled GRP/bombesin analog. By virtue of their potential to visualize tumor lesion GRP-R status and its effective downregulation after efficient treatment, radiolabeled GRP analogs have the potential to predict or rapidly assess response to GRP-R-targeted treatment modalities.

${ }^{99 \mathrm{~m}} \mathrm{Tc}-\mathrm{RP} 527$ consists of a targeting peptide derived from bombesin, linked at its $\mathrm{N}$-terminus via a linker group to a peptide sequence that chelates ${ }^{99 \mathrm{~m}} \mathrm{Tc}$. Unlabeled bombesin, uncomplexed RP527, and the structural mimic of ${ }^{99 \mathrm{~m} T c-}$ RP527-RP720 (ReO-RP527)—inhibit binding of ${ }^{125} \mathrm{I}-$ bombesin to GRP-R-expressing PC-3 and CF-PAC-1 cells (human prostate and pancreatic cancer cell lines, respectively) with similar mean 50\% inhibitory concentration values ranging from 1.6 to $5.5 \mathrm{nM}$. Additionally, ${ }^{99 \mathrm{~m} T c-}$ RP527 was rapidly and specifically bound and internalized by both PC-3 and CF-PAC-1 cells (5). The ligand has been shown to be safe and to exhibit good imaging characteristics $(6,7)$. To our knowledge, data on the relationship between the uptake of ${ }^{99 \mathrm{~m}} \mathrm{Tc}-\mathrm{RP} 527$ by tumor tissue versus GRP-R status assessed by a gold standard have not been reported previously. This study examined the relationship between ${ }^{99 \mathrm{~m}} \mathrm{Tc}-$ RP527 uptake in tumor lesions of patients with breast carcinoma and GRP-R expression as measured by immunohistochemistry (IHC).

\section{MATERIALS AND METHODS}

\section{Patients}

This study was approved by the Medical Ethics Committee of the University Hospital and performed according to Good Clinical Practice guidelines. Fourteen patients (mean age, 58.6 y; range, $37-75 \mathrm{y}$ ) were included in the study. All patients gave their written informed consent for participation in the study. Nine patients were referred because of a clinical diagnosis highly suggestive of breast 
carcinoma. These patients underwent ${ }^{99 \mathrm{~m}} \mathrm{Tc}-\mathrm{RP} 527$ scintigraphy on the day before biopsy or fine-needle biopsy or removal of the suspected mass. In addition to routine histopathologic analysis, slides of the primary lesion of these patients were stained for GRP-R. Another 5 patients with histologically proven and documented tamoxifen-resistant bone-metastasized breast carcinoma also underwent ${ }^{99 \mathrm{~m}}$ Tc-RP527 scintigraphy. In all 14 patients, routine staging examinations were performed within $1 \mathrm{wk}$ after 99mTc-RP527 scintigraphy. Staging was performed according the TNM classification criteria.

\section{Methods}

Radiopharmaceutical Synthesis. ${ }^{99 \mathrm{~m} T c-R P 527}$ labeling was performed using a kit formulation; $0.1 \mathrm{~mL}$ of $2 \mathrm{mM}$ stannous chloride, $0.1 \mathrm{~mL}$ of $60 \mathrm{mM}$ sodium gluconate, $1,850-2,035 \mathrm{MBq}$ of ${ }^{99} \mathrm{TcO}_{4}$ in $0.3 \mathrm{~mL}$ of $0.9 \%$ sodium chloride, and $0.5 \mathrm{~mL}$ of $0.9 \%$ sodium chloride were added to $100 \mu \mathrm{g}$ of RP527. After $35 \mathrm{~min}$ in a boiling water bath, the reaction mixture was allowed to cool down to room temperature and injected on a high-performance liquid chromatography system using an ethanol/water/acetic acid gradient. The radiolabeled peptide was collected at $45 \mathrm{~min}$, and the collected eluent was diluted with $10 \mathrm{~mL}$ of $0.9 \%$ sodium chloride. The overall yield of the radiosynthesis was about $30 \%$, with a radiochemical purity $\geq$ $90 \%$ and a specific activity $\geq 4.32 \mathrm{TBq} / \mu \mathrm{mol}$.

Scintigraphy. Subjects were positioned supine with their arms alongside their body. Whole-body images were performed using a triple-head $\gamma$-camera (Irix; Picker), equipped with low-energy, high-resolution, parallel-hole collimators. The energy peak was centered at $140 \mathrm{keV}$ with a $15 \%$ window. Whole-body (scan speed, $11.3 \mathrm{~cm} / \mathrm{min}$ ) and tomographic scans were acquired 5-6 h after injection. Localized SPECT acquisitions made of known tumor regions with a $120 \times 20 \mathrm{~s}$ acquisition over $360^{\circ}$ were reconstructed iteratively (ordered-subset expectation maximization algorithm; 2 iterations, 6 subsets) and postfiltered using a Butterworth filter (cutoff frequency, 0.8 cycle/cm; order, 8 ). The ratios of tumor to normal tissue ( $\mathrm{T} / \mathrm{N}$ ratios) were determined on summed tomographic slices by placing a region of interest (ROI) over the hottest part of the tumor (T) and an identically sized ROI over contralateral normal breast tissue $(\mathrm{N})$.

IHC. IHC was performed on formalin-fixed paraffin-embedded samples of the primary tumor held by the Department of Pathology of the Ghent University Hospital. Sections (4- $\mu$ m-thick) were mounted on SuperFrost microscope slides (Menzel-Glaser), which were deparaffinized in xylene and rehydrated in a downgraded series of ethanol. The GRP-R status of lymph nodes was not assessed as the nodes were not systematically available and also to avoid partial-volume-related discordances. After flushing in water, heat-induced antigen retrieval was performed for $20 \mathrm{~min}$ with a corresponding buffer (citrate buffer, $\mathrm{pH}$ 6.0), cooled down for 20 $\mathrm{min}$, and then flushed in water for $10 \mathrm{~min}$. Endogenous tissue peroxidase was blocked for 5 min with $\mathrm{H}_{2} \mathrm{O}_{2}$ (Dako). Slides were then incubated with a commercially available primary antihuman GRP-R antibody (Santa Cruz Biotechnology) diluted in $1 \%$ bovine serum albumin/Tris-buffered saline and incubated for $1 \mathrm{~h}$; the dilution factor was 1:10. After washing, the sections were incubated for $30 \mathrm{~min}$ with a labeled polymer-horseradish peroxidase antirabbit secondary antibody (Dako). The chromogen 3,3'-diaminobenzidine (Dako) was used for spectrophotometric visualization of the signal as brown staining. After washing, the sections were counterstained with hematoxylin.
Microscopic Analysis. The intensity and amount of GRP-Rpositive tumor cells in the immunoreaction were determined independently by 2 pathologists who were unaware of the scintigraphic results. The percentage of tumor cells that were GRPR-R positive was scored as follows: $0 \%$, score $0 ; 0 \%-20 \%$, score 1 ; $20 \%-40 \%$, score $2 ; 40 \%-60 \%$, score $3 ; 60 \%-80 \%$, score 4 ; and $80 \%-100 \%$, score 5 . Intensities of staining were classified from 0 (absent staining) to 4 (high staining). A score of 1, 2, or 3 corresponds to very low, low, and intermediate intensity of staining, respectively. For each tumor, a value designated as the $\mathrm{H}$ score was than obtained by multiplying the intensity score by the percentage score. This methodology was shown previously to be highly reproducible (intraclass correlation analysis for intra- and interobserver variability: $r=0.94[P=0.001]$ and $r=0.9[P=0.001]$, respectively) (8).

Routine immunohistochemical assessment of estrogen receptor (ER) and progesterone receptor (PR) status was performed using commercially available antibodies.

\section{RESULTS}

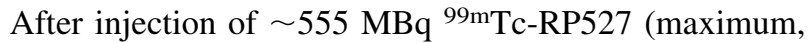
$3 \mathrm{ng} / \mathrm{kg}$ per subject), no adverse or subjective effects were noted in any of the subjects. The whole-body and tomographic images obtained show a diffuse and heterogeneous uptake of radioactivity in the normal breast tissue, limited to the central, periaureolar glandular part of the breast. Imaging results of 6 of the patients included were reported previously (6).

Results of the 9 patients referred because of a clinical diagnosis highly suggestive of breast carcinoma are shown in Table 1. All 9 patients with suspected breast lesions were tumor positive on histology. Four of 9 patients were ER and PR positive and 2 of 9 patients were solely ER positive. Primary tumor ${ }^{99 \mathrm{~m}}$ Tc-RP527 uptake was clearly depicted in 8 of 9 patients. In these 8 patients, involved lymph nodes and part of the distant metastases limited to the bone (occurring in only 1 patient), when present, also showed ${ }^{99 \mathrm{~m}}$ Tc-RP527 uptake (Figs. 1 and 2). Tumor-to-background ratios of involved lymph nodes and bone metastases in these patients ranged from 2 to 20 (mean, 10.3). In ${ }^{99 m}$ Tc-RP527-positive patients, $\mathrm{T} / \mathrm{N}$ ratios of the primary tumor derived from tomographic images ranged from 1 to 14 (mean, 6.3). GRP-R staining was positive in all 9 patients but to a variable degree: $\mathrm{H}$ scores ranged from 2 to 15 (mean, 8.4). Staining was cytoplasmatic as well as membranous and was more pronounced in the well-differentiated parts of the tumors. There was, however, no relationship between the global degree of differentiation and obtained $\mathrm{H}$ scores. Histologic $\mathrm{H}$ scores were significantly correlated with $\mathrm{T} / \mathrm{N}$ ratios (Spearman rank; $r=0.929, P=0.0001$ ) (Fig. 3) (8).

Osseous involvement was not visualized by means of ${ }^{99 m}$ Tc-RP527 scintigraphy in any of the 5 bone-metastasized breast carcinoma patients.

\section{DISCUSSION}

Our data show ${ }^{99 \mathrm{~m}} \mathrm{Tc}-\mathrm{RP} 527$ uptake in untreated primary breast carcinoma and related metastases, but not in metas- 
TABLE 1

Clinical, Pathologic, and Scintigraphic Findings in Patients Presenting with Primary Untreated Breast Carcinoma

\begin{tabular}{lclllrrrrr}
\hline Patient no. & Age $(\mathrm{y})$ & Histology & Size & TNM stage & ER & PR & GRP-R H score & Diff. & T/N ratio \\
\hline 1 & 37 & Inv. lob. & 6.0 & T3 N1 M0 & 0 & 0 & 15 & Poor & 9.5 \\
2 & 46 & Inv. lob. & 5.5 & T3 N2 M0 & 0 & 0 & 2 & Moderate & 2.1 \\
3 & 63 & Inv. lob. & 1.5 & T1 N0 M0 & 90 & 90 & 4 & Poor & 1.8 \\
4 & 69 & Inv. duct. & 4.0 & T2 N1 M0 & 75 & 60 & 6 & Moderate & 4.5 \\
5 & 44 & Inv. duct. & 5.4 & T3 N1 MO & 0 & 0 & 8 & Moderate & 4.6 \\
6 & 41 & Inv. lob. & 3.5 & T2 N1 M0 & 90 & 80 & 12 & Poor & 11 \\
7 & 75 & Inv. duct. & 5.6 & T4 N1 M0 & 95 & 0 & 12 & Poor & 8.2 \\
8 & 51 & Inv. lob. & 3.0 & T2 N3 M1 & 90 & 50 & 2 & Moderate & 1 \\
9 & 65 & Inv. lob. & 4.0 & T4 N3 M1 & 98 & 5 & 15 & Moderate & 14
\end{tabular}

Size = largest-diameter primary tumor; TNM = tumor-node-metastasis stage; $\mathrm{ER}=\mathrm{ER}$ status; $\mathrm{PR}=\mathrm{PR}$ status; GRP-R H score $=$ histologic GRP-R score; Diff. = degree of differentiation of primary tumor; $\mathrm{T} / \mathrm{N}$ ratio = ratio of tumor to normal tissue; Inv. lob. = invasive lobular carcinoma; Inv. duct. = invasive ductal carcinoma.

tases of previously treated patients, as well as ubiquitous and heterogeneous ${ }^{99 m}$ Tc-RP527 uptake in nonneoplastic breast tissue.

Expression of GRP-R in primary human breast carcinoma has been studied previously by methodologies other than scintigraphy or IHC. Using receptor-binding techniques on tumor homogenates, Halmos et al. found that 33\% (33/100) of primary breast carcinomas were GRP-R positive (9). On the other hand, using in vitro autoradiography, Gugger et al. reported a $62 \%(44 / 71)$ incidence of GRP-Rs in primary breast carcinomas (10). In the series presented, 8 of $9(88 \%)$ primary breast carcinomas were GRP-R positive as measured by in vivo ${ }^{99 \mathrm{~m}} \mathrm{Tc}-\mathrm{RP} 527$ scintigraphy, and all primary tumors studied proved to be positive on IHC. It is important to note that semiquantitative histologic GRP-R scores were significantly correlated with $\mathrm{T} / \mathrm{N}$ ratios. The different incidence numbers found between our series and those by Halmos et al. and Gugger et al. likely relate to differences in patient inclusion and methodology. Of interest, if the primary tumor was GRP-R positive as assessed by ${ }^{99 \mathrm{~m}} \mathrm{Tc}-$ RP527 scintigraphy, involved lymph nodes were also positive and easily depictable, even if a single lymph node was involved. This finding is in agreement with the data by Gugger et al. showing a high GRP-R density and homogeneous distribution in axillary lymph-node metastases and the lack of GRP-Rs in surrounding lymphoreticular tissue (high
FIGURE 1. (A and B) Faint GRP-R staining of breast carcinoma cells in patient $8(\mathrm{~A})$, indicated by small arrows, and lack of $99 \mathrm{mTc}-\mathrm{RP} 527$ uptake in the same patient's breast tumor on transaxial slices (B). (C and D) Pronounced GRP-R staining of breast carcinoma cells in patient $1(\mathrm{C})$, indicated by small arrows, and high uptake of $99 \mathrm{mTc}-\mathrm{RP} 527$ uptake in the same patient's breast tumor on transaxial slices (D).

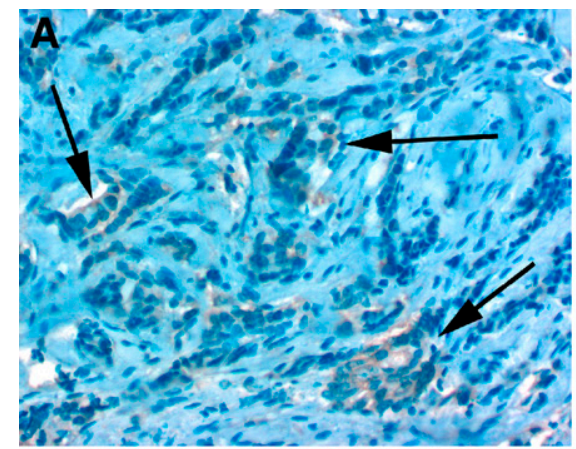

B
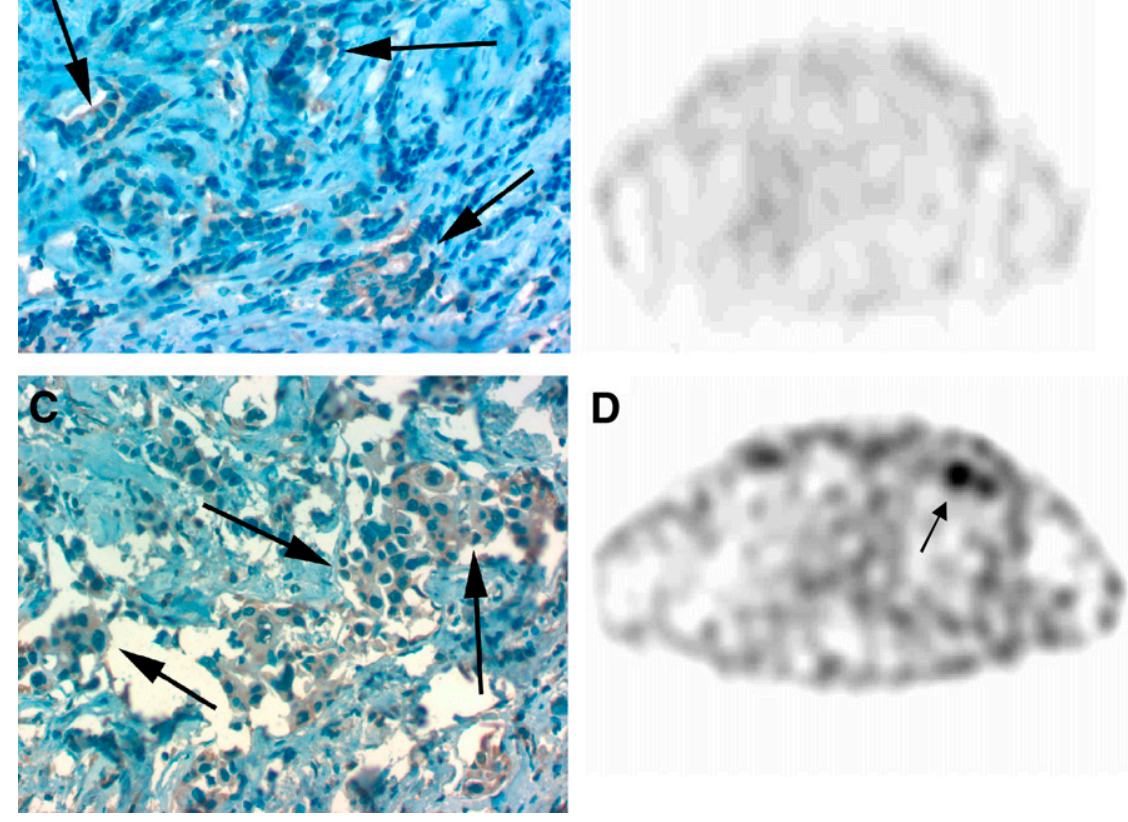


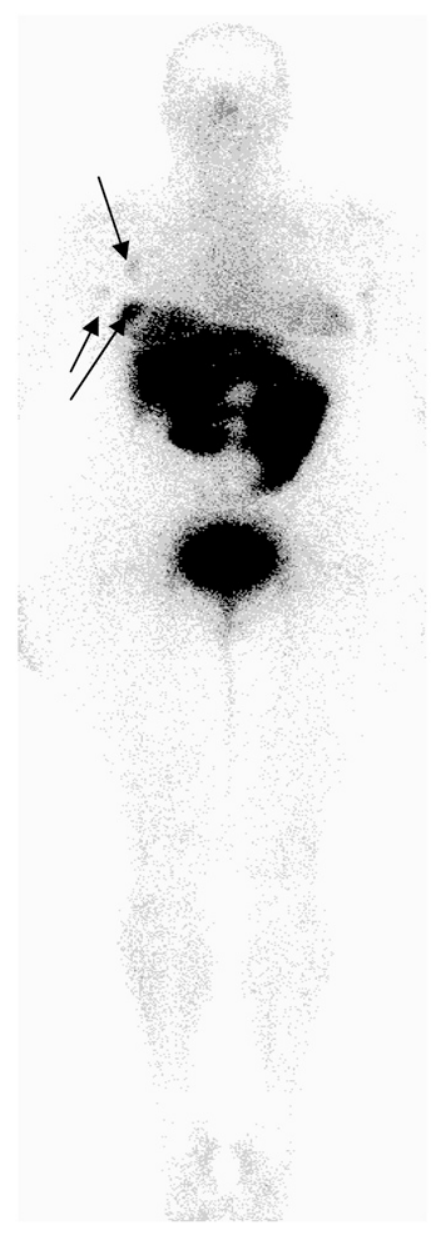

FIGURE 2. Anterior wholebody scan of patient 9 documenting 99mTc-RP527 uptake in primary breast tumor, involved axillary lymph nodes, and a rib metastasis (indicated by arrows).

tumor-to-background ratio) (10). Finally, in the 1 patient presenting with a primary breast carcinoma and osseous involvement, both primary but only half of the metastatic bone

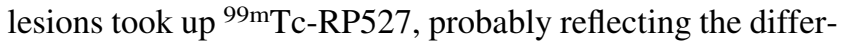

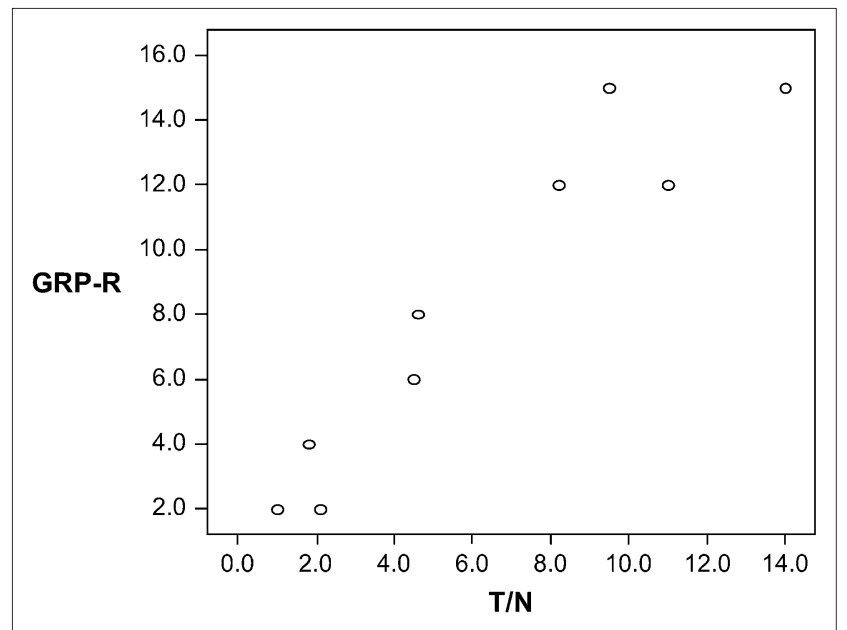

FIGURE 3. Scatter plot shows relationship between 99mTcRP527 tumor-to-background ratios and GRP-R H score. ence in clonal origin — with distinct biologic parameters—of metastatic lesions.

In the 5 patients with tamoxifen-resistant osseous disease, none of the known sites of osseous involvement were positive on ${ }^{99 \mathrm{~m}} \mathrm{Tc}-\mathrm{RP} 527$ scintigraphy. The absence of uptake in these less-differentiated tumors when compared with untreated primary tumors and the more-pronounced expression on well-differentiated areas of tumor as demonstrated by IHC in this study supports the notion that apart from its known mitogenic and growth-stimulating activivity (2), GRP-R could also act as a morphogen or differentiation factor in breast carcinoma. A role for GRP-R as a morphogen has been reported previously in other types of tumors (11).

Finally, Gugger et al. described a ubiquitous heterogeneous GRP-R expression in normal breast ductules and lobules, possibly related to a heterogenous innervation pattern of the glands and lobules, assuming that GRP plays a neurotransmitter role in the breast, as it does in the gastrointestinal tract (10). As the sample size containing nonneoplastic breast tissue was often small, the authors suggested that the percentage of receptor heterogeneity may not be representative for the whole breast. The ${ }^{99 \mathrm{~m} T c-R P 527 \mathrm{im}-}$ ages show that the heterogeneous GRP-R expression to be limited to the central, glandular part of the breast. The reason for this heterogeneity and the relative high incidence when compared with other hormone receptors-for example, ER and PR (Table 1) - warrants further investigation. Possibly GRP-R, similar to ER and PR, may be involved in the regulation of mammary cell proliferation and differentiation.

During the past $10 \mathrm{y}$, several cytotoxic peptide hormone analogs of GRP and bombesin, as well as $\beta$-emitterradiolabeled bombesin derivatives, have been developed for the purpose of improving outcome in patients with GRP-R-expressing malignancies such as breast carcinoma $(12,13) .{ }^{99 \mathrm{~m} T c-R P 527}$ might allow for selection of those patients that will ultimately benefit from these novel treatment options. The advantages and disadvantages of ${ }^{99 \mathrm{~m}} \mathrm{Tc}-\mathrm{RP} 527$ SPECT versus PET with newly developed positron-emitterradiolabeled bombesin derivatives warrant further exploration (14).

\section{CONCLUSION}

In this pilot study, ${ }^{99 \mathrm{~m}} \mathrm{Tc}-\mathrm{RP} 527$ imaging results obtained in breast carcinoma patients were significantly correlated with results obtained by means of IHC.

\section{REFERENCES}

1. Van de Wiele C, Dumont F, van Belle S, Slegers G, Peers SH, Dierckx RA. Is there a role for agonist gastrin releasing peptide receptor radioligands in tumour imaging? Nucl Med Commun. 2001;22:5-15.

2. Engel JB, Schally AV, Dietl J, Rieger L, Honig A. Targeted therapy of breast and gynecological cancers with cytotoxic analogues of peptide hormones. Mol Pharm. 2007;4:652-658.

3. Van Eijck CH, Kwekkeboom DJ, Krenning EP. Somatostatin receptors in breast cancer. Q J Nucl Med. 1998;42:18-25.

4. Virgolini I, Raderer M, Kurtaran A, et al. ${ }^{123}$ I-Vasoactive intestinal peptide (VIP) receptor scanning: update of imaging results in patients with adenocarcinomas 
and endocrine tumors of the gastrintestinal tract. Nucl Med Biol. 1996;23:685692.

5. Hofmann TJ, Simpson SD, Smith CJ, et al. Accumulation and retention of ${ }^{99 \mathrm{~m}} \mathrm{Tc}$ RP527 by GRP receptor expressing tumors in SCID mice [abstract]. J Nucl Med. 1999;40(suppl):104P.

6. Van de Wiele C, Dumont F, Van den Broecke R, et al. Technetium99m RP527, a GRP analogue for visualisation of GRP receptor expressing malignancies: a feasibility study. Eur J Nucl Med. 2000;27: 1694-1699.

7. Van de Wiele C, Dumont F, Dierckx R, et al. Biodistributaion and dosimetry of ${ }^{99 \mathrm{~m} T c-R P 527}$, a gastrin-releasing peptide (GRP) agonist for the visualization of GRP receptor expressing malignancies. J Nucl Med. 2001;42:17221727.

8. Loose D, Signore A, Staelens L, et al. ${ }^{123}$ I-Interleukin-2 uptake in squamous cell carcinoma of the head and neck. Eur J Nucl Med Mol Imaging. 2007, October 10, 2007 [Epub ahead of print].
9. Halmos G, Wittliff JL, Schally AV. Characterization of bombesin/gastrin releasing peptide receptors in human breast cancer and their relationship to steroid receptor expression. Cancer Res. 1995;55:280-287.

10. Gugger M, Reubi JC. Gastrin releasing peptide receptors in non-neoplastic and neoplastic human breast. Am J Pathol. 1999;155:2067-2076.

11. Jensen JA, Carroll RE, Benya RV. The case for gastrin-releasing peptide acting as a morphogen when it and its receptor are aberrantly expressed in cancer. Peptides. 2001;22:689-699.

12. Nagy A, Schally A. Targeting cytotixc conjugates of somatostatin, luteinizing hormone-releasing hormone and bombesin to cancers expressing their receptors: a "smarter" chemotherapy. Curr Pharm Des. 2005;11:1167-1180.

13. Panigone S, Nunn A. Lutetium-177-labeled gastrin releasing peptide receptor binding analogs: a novel approach to radionuclide therapy. $Q \mathrm{~J} \mathrm{Nucl} \mathrm{Med} \mathrm{Mol}$ Imaging. 2006;50:310-321.

14. Zhang X, Cai W, Cao F, et al. ${ }^{18} \mathrm{~F}$-Labeled bombesin analogs for targeting GRP receptor-expressing prostate cancer. J Nucl Med. 2006;47:492-501. 